\title{
Hongos formadores de micorriza arbuscular asociados a plantaciones de Ochroma pyramidale (Cav. ex Lam.) Urb. (balsa) en el Trópico Húmedo Ecuatoriano
}

\section{Arbuscular mycorrhizal fungi associated to Ochroma pyramidale (Cav. ex Lam.) Urb. (balsa) in the Ecuadorian Humid Tropic}

\author{
Belezaca Pinargote Carlos ${ }^{1 *}$, Diana Calle Gill ${ }^{1}$, Oscar Prieto Benavides ${ }^{1}$, Rolando López Tobar ${ }^{1}$, \\ Edison Solano Apuntes ${ }^{1}$, Fabricio Meza Bone ${ }^{1}$ \\ ${ }^{1}$ Universidad Técnica Estatal de Quevedo, Carrera de Ingeniería Forestal, Quevedo, Ecuador. \\ *Autor para correspondencia: cbelezaca@uteq.edu.ec
}

\begin{abstract}
Resumen
$E_{g}^{1}$ objetivo de esta investigación fue conocer los géneros de hongos formadores de micorriza arbuscular (HMA) asociados a árboles de Ochroma pyramidale (balsa) en plantaciones y su grado de infección radicular. Se estudiaron seis plantaciones de 1, 2, 3, 4, 5.5 y 7 años de edad ubicadas en la zona central del Trópico Húmedo Ecuatoriano (THE), en cada una de ellas se delimitaron tres parcelas de 500 $\mathrm{m}^{2}$. En cada parcela se colectaron 3 muestras de suelo (aproximadamente $1 \mathrm{~kg}$ ), y raicillas a una profundidad entre $0-15 \mathrm{~cm}$ para determinar el porcentaje de colonización micorrízica. El aislamiento e identificación de los HMA a nivel de género y la estimación de colonización micorrizica se realizó mediante la metodología de tamizado, decantación en húmedo con centrifugación, y despigmentación - tinción de raíces. Se aislaron e identificaron cuatro géneros de HMA: Glomus, Acaulospora, Scutellospora, y Gigaspora, no obstante, predominó el género Glomus en el suelo de todas las plantaciones estudiadas con poblaciones que estuvieron entre 27 y 169 esporas por 100 gamos de suelo seco, siendo la plantación de 4 años donde se encontró la mayor población, mientras que los demás géneros mostraron baja representatividad en todas las edades. Se detectó baja colonización micorrízica radicular en las plantaciones de balsa estudiadas, con rangos de $0.28 \%$ a $0.65 \%$, porcentajes de colonización menores a los reportados para otras especies leñosas del THE.
\end{abstract}

Palabras clave: Acaulospora, Gigaspora, Glomus, Scutellospora, suelo.
Rec.: 11.10.2018. Acept.: 05.06.2019. Publicado el 31 de junio de 2019
$T$ The objective of this investigation was to determine the genera of arbuscular mycorrhizal fungi (AMF) associated with Ochroma pyramidale (balsa) trees and their degree of root infection. Where studied six plantations of $1,2,3,4,5.5$ and 7 years old, located in the central zone of the Ecuadorian Humid Tropic (EHT), in each of them three parcels of $500 \mathrm{~m}^{2}$ were delimited. From each plot, 3 soil samples (approximately $1 \mathrm{~kg}$ ) and rootlets at a depth between $0-15 \mathrm{~cm}$ were collected to determine the percentage of mycorrhizal colonization. Isolation and identification of AMF at genus level and the estimation of mycorrhizal colonization was carried out by means of sieving methodology, wet decantation with centrifugation, and depigmentation - roots staining. Four genera of AMF were isolated and identified: Glomus, Acaulospora, Scutellospora, and Gigaspora, however, the genus Glomus predominated in the soil of all plantations. The populations were between 27 and 169 spores per 100 grams of dry soil. Four year old plantation contained he largest population, while other genera showed low representativeness in all ages. Low root mycorrhizal colonization was detected in the plantations balsa studied, with ranges from $0.28 \%$ to $0.65 \%$, colonization rates lower than those reported for other woody species.

Keywords: Acaulospora, Gigaspora, Glomus, Scutellospora, soil. 


\section{Introducción}

$\mathrm{L}$ a especie forestal Ochroma pyramidale (Cav. ex Lam.) Urb. (balsa), es nativa del sur de México, Centroamérica, el Caribe y Suramérica, mundialmente conocida por las cualidades de su madera de baja densidad (González et al., 2010). Ecuador es considerado un referente y exportador mundial de la madera de esta especie (Jiménez-Romero et al., 2017), donde hasta el año 2015 la balsa representaba el 19\% de las plantaciones establecidas en el país con 9928 hectáreas, ubicándola como la tercera especie forestal más plantada (MAGAP, 2016).

De acuerdo a la distribución natural en Ecuador, la balsa predomina en zonas tropicales de la región oriental, occidental y estribaciones de la cordillera de Los Andes (Jiménez-Romero et al., 2017), y considerando que las interacciones biológicas son muy dinámicas en las regiones tropicales (Wright, 2001), es probable que la balsa igual a muchas especies vegetales haya coevolucionado junto a otros organismos. En este sentido, los hongos formadores de micorriza arbuscular (HMA) forman estrechas relaciones simbióticas con alrededor del $70 \%$ de las plantas vasculares del planeta (Lekberg et al., 2015) y $O$. pyramidale no sería la excepción.

Se conocen reportes de HMA asociados a plantaciones de varias especies leñosas en el trópico húmedo ecuatoriano (THE), como Tectona grandis L. f. (Contreras, 2016), Gmelina arborea Roxb. (Meza et al., 2017) y sistemas agroforestales tradicionales basados en Theobroma cacao L. (Prieto et al., 2011 y 2012a), sin embargo, solo existe un estudio de este grupo de hongos en suelos de plantaciones comerciales de $O$. pyramidale realizado por Prieto et al., (2012b), pese que la asociación HMA-vegetales genera beneficios mutuos a través de un sistema de transferencia bidireccional, basado en el flujo de nutrientes inorgánicos, especialmente de aquellos con escasa movilidad ( $\mathrm{P}, \mathrm{Zn}, \mathrm{Cu}, \mathrm{K}$, etc.), desde suelo a la planta y compuestos orgánicos como carbohidratos de la planta hacia el hongo (Peterson et al., 2004; Van Der Heijden et al., 2015), además, generan resistencia frente al ataque de patógenos radiculares (Huang et al., 2003); tolerancia ante la presencia de metales pesados en el suelo (Miransari, 2017), estrés hídrico (Wu \& Zou, 2017), condiciones extremas de $\mathrm{pH}$ del suelo (Seguel et al., 2016).

En este sentido, la calidad sanitaria y rendimiento de las plantaciones de balsa en el THE, hace sospechar que esta especie forestal formaría asociaciones simbióticas con uno o más géneros y/o especies de HMA nativos, sin embargo, lo poco que se sabe al respecto son solo especulaciones basadas en experiencias con otras especies forestales. La presente investigación planteó conocer que géneros de HMA se encuentran asociados a árboles de $O$. pyramidale en plantaciones y su grado de infección radicular.

\section{Materiales y métodos}

\section{Áreas de estudio y establecimiento de parcelas experimentales}

Se seleccionaron 6 plantaciones de $O$. pyramidale con edades de 1, 2, 3, 4, 5.5 y 7 años, y 3 ciclos de rotación, ubicadas en la zona central del THE (recintos: El Vergel, Delia María, Guapara, y Santa Rosa). (Figura 1; Cuadro 1). En cada plantación se delimitaron tres

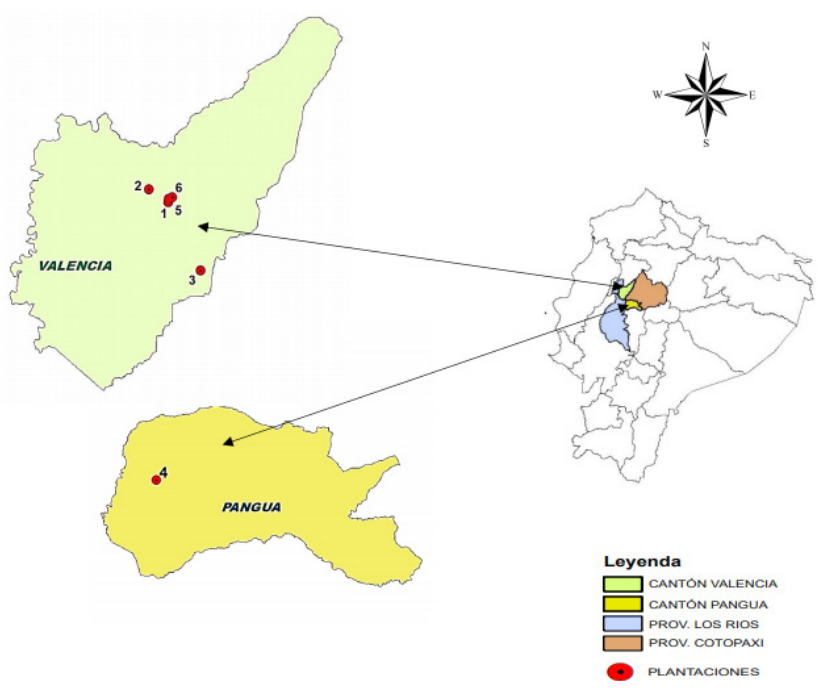

Figura 1. Distribución y ubicación geográfica de las plantaciones de Ochroma pyramidale estudiadas en la zona central del Trópico Húmedo Ecuatoriano. 
Cuadro 1. Codificación, densidad y coordenadas geográficas de las plantaciones de Ochroma pyramidale estudiadas en la zona central del Trópico Húmedo Ecuatoriano.

\begin{tabular}{|c|c|c|c|c|c|c|c|c|}
\hline \multirow{2}{*}{ No. } & \multirow{2}{*}{ Provincia } & \multirow{2}{*}{ Cantón } & \multirow{2}{*}{ Sector } & \multicolumn{2}{|c|}{$\begin{array}{c}\text { Plantaciones } \\
\text { (hacienda) }\end{array}$} & \multirow{2}{*}{$\begin{array}{c}\text { Densidad } \\
\text { (árboles/ha) }\end{array}$} & \multicolumn{2}{|c|}{ Coordenadas } \\
\hline & & & & Nombre & $\begin{array}{l}\text { Edad } \\
\text { (años) }\end{array}$ & & Latitud S & Longitud O \\
\hline 1 & Los Ríos & Valencia & El Vergel & PB 22 & 1 & 780 & $0^{\circ} 46^{\prime} 02^{\prime \prime}$ & $79^{\circ} 17^{\prime} 31^{\prime \prime}$ \\
\hline 2 & Los Ríos & Valencia & El Vergel & CEB 04 & 2 & 453 & $0^{\circ} 45^{\prime} 04^{\prime \prime}$ & $79^{\circ} 18^{\prime} 54^{\prime \prime}$ \\
\hline 3 & Los Ríos & Valencia & Delia María & FC 02 & 3 & 326 & $0^{\circ} 50^{\prime} 59^{\prime \prime}$ & $79^{\circ} 15^{\prime} 12^{\prime \prime}$ \\
\hline 4 & Cotopaxi & Pangua & Guapara & PB 49 & 4 & 613 & $1^{\circ} 01^{\prime} 48^{\prime \prime}$ & $79^{\circ} 16^{\prime} 12^{\prime \prime}$ \\
\hline 5 & Los Ríos & Valencia & El Vergel & PB 22 & 5.5 & 380 & $0^{\circ} 45^{\prime} 46^{\prime \prime}$ & $79^{\circ} 17^{\prime} 29^{\prime \prime}$ \\
\hline 6 & Los Ríos & Valencia & El Vergel & FC 06 & 7 & 400 & $0^{\circ} 45^{\prime} 39^{\prime \prime}$ & $79^{\circ} 17^{\prime} 14^{\prime \prime}$ \\
\hline
\end{tabular}

parcelas de $500 \mathrm{~m}^{2}$, dentro de las cuales y por una solo vez se determinaron las variables dasométricas altura total y DAP (diámetro a la altura del pecho a $1.30 \mathrm{~m}$ sobre el nivel del suelo) en los árboles de balsa en pie.

\section{Recolección de material biológico}

En cada plantación se recolectaron 3 muestras de suelo de aproximadamente $1 \mathrm{~kg}$, y raicillas (para determinar el porcentaje de colonización micorrízica presente), a una profundidad comprendida entre 0 $-15 \mathrm{~cm}$. Tanto el suelo y las raicillas se depositaron dentro de fundas plásticas rotuladas, e inmediatamente se introdujeron en cajas refrigeradas (hieleras) con el propósito de evitar modificaciones significativas en la biología del suelo por aumentos bruscos de temperatura.

\section{Aislamiento, identificación y conteo de HMA}

Se empleó la metodología propuesta por Gerderman $\&$ Nicholson (1963) con modificaciones, que consistió en aislar esporas de HMA mediante el tamizado del suelo y decantación en húmedo. Para la estimación del número de esporas por gramo de suelo seco, se pesaron $100 \mathrm{~g}$ de suelo húmedo y secaron en una estufa a 65 ${ }^{\circ} \mathrm{C}$ durante 72 horas. Posteriormente se registró el peso del suelo seco y mediante una regla de tres simple se calculó el número de esporas por gramo de suelo seco. La identificación de los HMA se realizó a nivel de morfoespecies con la ayuda un microscopio y el apoyo de claves taxonómicas (Sieverding, 1991; Brundrett et al., 1996; INVAN, 2016).

\section{Porcentaje de colonización micorrízica}

Para su estimación se aplicó la metodología propuesta por Philips y Hayman (1970) y Giovannetti y Mosse (1980) con modificaciones, la cual se basa en la decoloración de raicillas con $\mathrm{KOH}$ al $10 \%$ y $\mathrm{H}_{2} \mathrm{O}_{2}$ al $3 \%$, acidificación con $\mathrm{HCl} 1 \mathrm{~N}$, y tinción de las raicillas con Azul de Tripano al $0.05 \%$ en lactoglicerol. Por cada muestra se realizaron montajes de 30 segmentos de raíces en láminas portaobjetos adicionando gotas de glicerol $(50 \%)$. Las observaciones se hicieron por triplicado (3 repeticiones) en un microscopio a 40x.

La frecuencia (\%) de colonización radicular (micelio, arbúsculos y vesículas) se determinó considerando los segmentos colonizados y no colonizados, mediante la relación del total de segmentos colonizados con respecto a los segmentos totales evaluados.

\section{Análisis estadístico}

Los datos obtenidos se analizaron empleando herramientas de estadística descriptiva. Para establecer si existían o no diferencias estadísticas en la densidad de hongos micorrízicos, y colonización radicular entre las diferentes edades de las plantaciones de balsa, los datos se sometieron a un análisis de varianza (ANOVA) con un nivel de significancia de 95\% $(P<0.05)$, previa comprobación de los supuestos de normalidad y homocedasticidad de varianzas. Posteriormente se aplicó la prueba LSD (mínima diferencia significativa), con un nivel de significancia del 95\% $(P<0.05)$. Para el efecto se empleó el paquete estadístico SYTAT 11 versión para Windows.

\section{Resultados}

\section{Variables dasométricas en plantaciones estudiadas}

En cuanto a las variables altura y DAP, los incrementos promedio fueron progresivos en función a la edad, con $10.2 \mathrm{~m}$ de altura y $17.0 \mathrm{~cm}$ de DAP para la plantación de 1 año, llegando a alcanzar los $24.3 \mathrm{~m}$ de altura y 30.0 de DAP en los árboles de la plantación de 7 años. No obstante, se detectó una excepción en los 
Tabla 2. Variables dasométricas evaluadas en seis plantaciones de $O$. pyramidale (balsa) de diferentes edades en la zona central del Trópico Húmedo Ecuatoriano.

\begin{tabular}{|c|c|c|c|c|c|c|c|c|c|}
\hline \multirow{2}{*}{ No. } & \multicolumn{2}{|c|}{ Plantaciones (hacienda) } & \multirow{2}{*}{$\begin{array}{c}\text { Árboles } \\
\mathrm{ha}^{-1}\end{array}$} & \multicolumn{3}{|c|}{ Alturas (m) } & \multicolumn{3}{|c|}{ DAP (cm) } \\
\hline & Nombre & Edad (años) & & Promedio & Máxima & Mínima & Promedio & Máximo & Mínimo \\
\hline 1 & $P B-22$ & 1 & 780 & $10.2 \pm 0.48 *$ & 11.0 & 9.0 & $17.0 \pm 1.66 *$ & 21.3 & 11.0 \\
\hline 2 & CEB - 04 & 2 & 453 & $17.4 \pm 1.50$ & 19.5 & 14.0 & $19.3 \pm 2.83$ & 27.1 & 12.4 \\
\hline 3 & $\mathrm{FC}-02$ & 3 & 326 & $24.0 \pm 2.52$ & 28.5 & 19.0 & $23.5 \pm 3.19$ & 31.8 & 17.2 \\
\hline 4 & $\mathrm{~PB}-49$ & 4 & 613 & $22.6 \pm 1.69$ & 26.0 & 17.5 & $28.8 \pm 5.00$ & 42.2 & 18.5 \\
\hline 5 & $\mathrm{~PB}-22$ & 5.5 & 380 & $27.8 \pm 1.86$ & 33.0 & 22.0 & $31.5 \pm 4.05$ & 45.2 & 22.9 \\
\hline 6 & $\mathrm{FC}-06$ & 7 & 400 & $34.3 \pm 3.64$ & 41.0 & 24.0 & $39.0 \pm 7.05$ & 56.5 & 26.1 \\
\hline
\end{tabular}

* Valores corresponden a promedios de 3 parcelas con su respectiva desviación estándar.

árboles de balsa de la plantación de 4 años (613 árboles $\mathrm{ha}^{-1}$ ) quienes alcanzaron una altura promedio de $22.6 \mathrm{~m}$, frente a la alcanzada por los árboles de 3 años con $24.0 \mathrm{~m}$, lo cual representó una disminución de 1.4 m (Cuadro 2).

\section{Identificación y densidad de HMA}

En los suelos de las plantaciones de balsa estudiadas se aislaron e identificaron cuatro géneros de HMA: Glomus, Acaulospora, Scutellospora, y Gigaspora. Se detectaron diferencias estadísticas significativas en el número promedio de esporas de cada género de HMA (esporas $100 \mathrm{~g}^{-1} \mathrm{ss}$ ) frente a las edades de las plantaciones. Para el caso de Glomus spp. $(F=9.79$; $P=0.00)$, la mayor densidad de esporas se encontró en la plantación de 4 años, y la menor en la de 5.5 años, con 169 y 30 esporas, respectivamente. Acaulospora sp. $(F=11.98 ; P=0.00)$ registró la mayor densidad en la plantación de 2 años con un total de 23 esporas, mientras que la menor densidad poblacional se registró en la plantación de 1 año, con tan solo 1 espora $100 \mathrm{~g}^{-1} \mathrm{ss}$. Scutellospora $\operatorname{sp}(F=10.73 ; P=0.00)$ presentó la mayor densidad poblacional en la plantación de 4 años, con un total de 2 esporas, mientras que en las plantaciones de 1 , 2, y 5.5 años no se registraron esporas. Para el caso de
Gigaspora sp. $(F=3.89 ; P=0.02)$ se evidenció la mayor densidad en la plantación de 3 años con un total de 6 esporas, mientras que en las plantaciones de 1 y 7 años no se detectó ninguna (Cuadro 3).

\section{Población total de HMA (esporas)}

Se detectaron diferencias estadísticas significativas $(F=10.36 ; P=0.00)$ entre la densidad total de esporas $100 \mathrm{~g}^{-1}$ ss de HMA (Glomus spp., Acaulospora spp., Scutellospora sp., y Gigaspora sp.) en las plantaciones de balsa de diferentes edades. Se determinó que en la plantación de cuatro años existió una mayor densidad de HMA por unidad de peso (185 esporas $100 \mathrm{~g}^{-1}$ ss), comportándose estadísticamente superior a las densidades encontradas en las plantaciones de las demás edades (Figura 2).

\section{Porcentaje de colonización micorrizica}

En la figura 3 se muestra el porcentaje de colonización micorrízica (densidad visual) obtenido en raicillas de $O$. pyramidale, donde se detectaron diferencias estadísticas significativas entre las edades de las plantaciones $(F=6.65 ; P=0.00)$, siendo las raicillas de las plantaciones de 3 y 7 años las que mostraron

Cuadro 3. Número de esporas (densidad) de HMA $100 \mathrm{~g}^{-1} \mathrm{ss}$ bajo el suelo de plantaciones de balsa de seis edades, ubicadas en la zona central del Trópico Húmedo Ecuatoriano. Valores corresponden a promedios de tres repeticiones con su respectivo error estándar. Letras iguales indican medias estadísticamente similares $(P<\mathbf{0 . 0 5})$.

\begin{tabular}{lccrr}
\hline \multirow{2}{*}{ Edad } & \multicolumn{5}{c}{ Géneros } \\
\cline { 2 - 5 } 1 año & $89 \pm 10.55 \mathrm{~b}$ & Acaulospora & Scutellospora & Gigaspora \\
\hline 2 años & $39 \pm 6.50 \mathrm{bc}$ & $23 \pm 0.46 \mathrm{a}$ & $0 \pm 0.00 \mathrm{c}$ & $0 \pm 0.00 \mathrm{c}$ \\
\hline 3 años & $27 \pm 2.06 \mathrm{c}$ & $3 \pm 0.22 \mathrm{~b}$ & $1 \pm 0.47 \mathrm{~b}$ & $5 \pm 1.18 \mathrm{ab}$ \\
\hline 4 años & $169 \pm 38.43 \mathrm{a}$ & $4 \pm 2.37 \mathrm{~b}$ & $2 \pm 0.39 \mathrm{a}$ & $6 \pm 2.84 \mathrm{a}$ \\
\hline 5.5 años & $30 \pm 8.40 \mathrm{c}$ & $4 \pm 0.81 \mathrm{~b}$ & $0 \pm 0.00 \mathrm{c}$ & $1.04 \mathrm{bc}$ \\
\hline 7 años & $66 \pm 8.78 \mathrm{bc}$ & $3 \pm 0.85 \mathrm{~b}$ & $1 \pm 0.15 \mathrm{~b}$ & $0 \pm 0.14 \mathrm{bc}$ \\
\hline
\end{tabular}




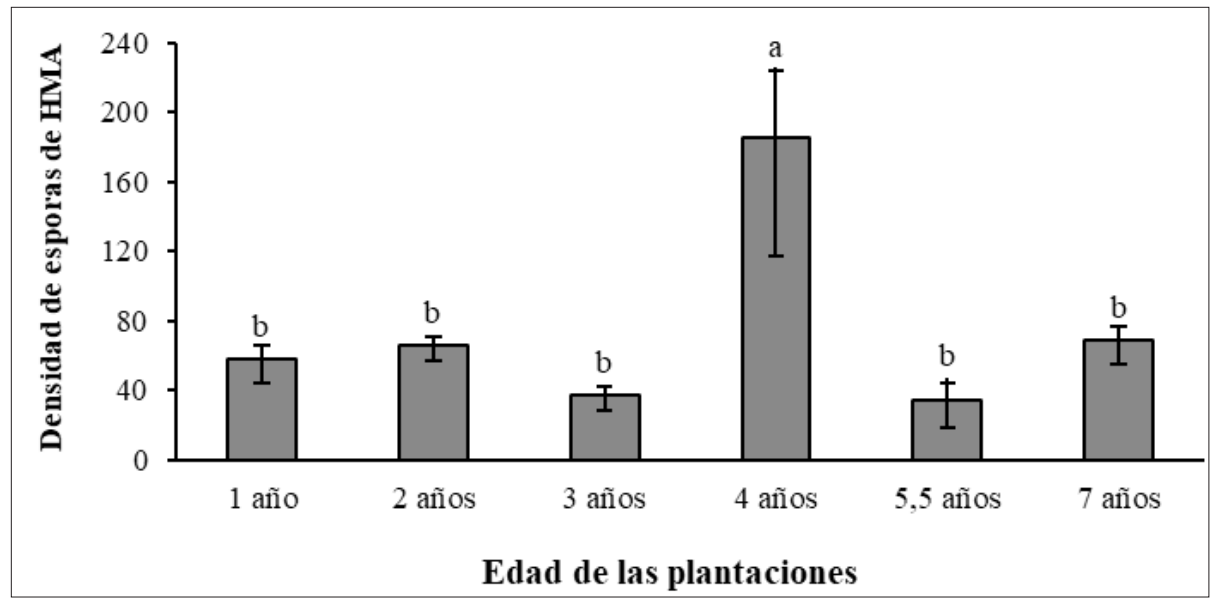

Figura 2. Número promedio total de esporas $100 \mathrm{~g}^{-1} \mathrm{ss}$ de HMA obtenidos en seis plantaciones de $\boldsymbol{O}$. pyramidale de diferentes edades. Valores corresponden a promedios de tres plantaciones con su respectivo error estándar y desviación estándar. Letras iguales indican medias estadísticamente similares $(\mathrm{P}<0.05)$.

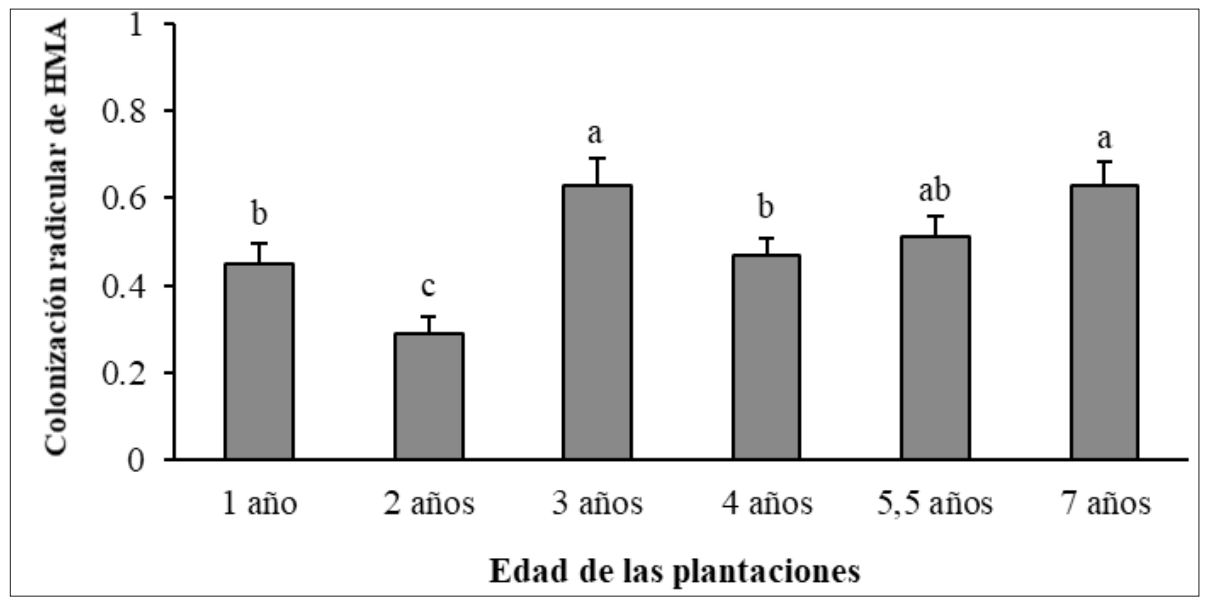

Figura 3. Porcentaje de colonización micorrízica (densidad visual) en raicillas de $\boldsymbol{O}$. pyramidale en plantaciones de diferentes edades. Valores corresponden a promedios de tres repeticiones (90 raicillas) por plantación con su respectivo error estándar y desviación estándar. Letras iguales indican medias estadísticamente similares $(\mathbf{P}<0.05)$.

un porcentaje mayor de colonización micorrízica en comparación a las de 1, 2, 4 y 5.5 años.

\section{Discusión}

$E^{1}$ 1 manejo silvicultural es una actividad clave para el mejoramiento de la producción y calidad de los bosques plantados (Nguyen et al., 2016). En este sentido $O$. pyramidale es una especie forestal que responde satisfactoriamente a esta estrategia cuando es aplicada oportunamente y de forma técnica (JiménezRomero et al., 2017). La densidad encontrada en las plantaciones de 1 año (780 árboles ha $\left.{ }^{-1}\right)$ está en los rangos empleados en plantaciones de balsa del Trópico Húmedo Ecuatoriano para esta edad, no obstante, en aquellas plantaciones de 2, 3, 4, 5.5 y 7 años de edad, si bien la densidad fue menor a la inicial, su disminución no estuvo acorde a la edad y turno final de aprovechamiento, tal es el caso de aquella que a los 4 años presentó una densidad de 613 árboles $\mathrm{ha}^{-1}$, efecto que se reflejó en árboles con alturas promedio de 22.6 $\mathrm{m}$, frente a aquellos árboles de 3 años, que pese a tener 
un año de edad menor, presentaron $1.4 \mathrm{~m}$ más $(24 \mathrm{~m})$ que la plantación de 4 años. Estos resultados muestran que un manejo silvicultural adecuado en balsa resulta en mejores respuestas de las variables dasométricas, caso contrario su potencial se reduce, comportamiento que constantemente es reportado por otros investigadores en varias especies forestales (Salazar-Zeledon, 2016).

Los géneros de HMA Glomus, Acaulospora, Scutellospora, y Gigaspora encontrados en suelos asociados a plantaciones de $O$. pyramidale de diferentes edades, han sido aislados e identificados desde plantaciones de otras especies forestales como Técntona grandis L. f. (Contreras, 2016), Gmelina arborea Roxb. (Meza et al., 2017) y sistemas agroforestales tradicionales basados en Theobroma cacao L. (Prieto et al., 2011 y 2012) en el Trópico Húmedo Ecuatoriano, destacando que los representantes de estos HMA se encuentran formando parte de la biodiversidad de los ecosistemas forestales de la región.

Si bien la mayor densidad poblacional de HMA asociada a suelos de plantaciones de balsa se encontró en la plantación de 4 años de edad (185 esporas 100 $\mathrm{g}^{-1} \mathrm{ss}$ ) muy por encima a la densidad encontrada en los suelos de plantaciones de 1, 2, 3, 5.5, y 7 años, con rangos entre 35 y 70 esporas $100 \mathrm{~g}^{-1} \mathrm{ss}$, estas densidades son cercanas a las 70 y 62 esporas $100 \mathrm{~g}^{-1}$ ss encontradas por Meza et al., (2017) en plantaciones de Gmelina arbórea (melina) de 1 y 3 años de edad respectivamente, en el THE. No obstante estas densidades están muy por debajo de los rangos 1030 a 2028 esporas 100 $\mathrm{g}^{-1}$ ss reportadas por Prieto et al., (2012) en sistemas agroforestales tradicionales basados en cacao del THE. Estos resultados podrían indicar que la densidad de HMA disminuye en plantaciones forestales monoespecíficas, pero se ven favorecidas en sistemas productivos con mayor diversidad vegetal leñosa, como es el caso de los sistemas agroforestales.

La mayor densidad de HMA encontrada en la plantación de 4 años podría indicar que a esta edad $O$. pyramidale es mayormente dependiente de la simbiosis con HMA, lo cual coincide con la edad en que los árboles incrementan significativamente su biomasa antes de su aprovechamiento al quinto año.

La baja densidad de colonización visual detectada en raíces de balsa $(0.30 \%-0.65 \%)$, en comparación a las reportadas por otros investigadores en diferentes especies vegetales leñosas del THE: Theobroma cacao L. $0.9 \%-3.5 \%$ (Prieto et al., 2012), Tectona grandes L. f. $1.3 \%-1.5 \%$ (Contreras 2016), Gmelina arbórea Roxb. 1.7\% - 1.9\%, (Meza et al., 2017), probablemente estaría asociada a la baja densidad de esporas de HMA encontrada en los suelos de las plantaciones de balsa estudiadas. Esta variabilidad interespecífica en la colonización radicular seguramente está asociada al tipo de especie vegetal (Peterson et al., 2004) sin embargo, la baja colonización micorrizica en las plantaciones de balsa de diferentes edades, podría indicar que es un fenómeno dominante y recurrente, producto de su coevolución, sin que este comportamiento afecte el normal crecimiento de la especie forestal.

\section{Conclusiones}

$E^{n}$ n suelos de plantaciones de $O$. pyramidale (balsa) habitan géneros de los HMA Glomus, Acaulospora, Scutellospora, y Gigaspora formando simbiosis con los sistemas radiculares de la especie forestal, y la densidad de colonización radicular, pese a ser baja, fue superior en plantaciones de 3 y 5 años de edad. En la plantación de 4 años se detectó una tendencia a presentar la mayor densidad poblacional de HMA.

\section{Agradecimientos}

Al proyecto de investigación FOCICYT-UTEQPFOC-3-1-2016, y al Ing. Marcelino Guachambala Cando, Gerente de Investigación y Desarrollo de la empresa 3A COMPOSITES PLANTABAL S.A.

\section{Bibliografía}

Brundrett, M.; Bougher, B.; Dell, T.; Grove \& N. Malajczuk. 1996. Working whit mycorrhizas in forestry and agriculture. Camberra, Australia. 374 p.

Contreras, J. 2016. Hongos micorrízicos de plantaciones de teca (Tectona grandis) y su potencial como biofertilizantes en plántulas a nivel de vivero. Tesis de Magister en Manejo y Aprovechamiento Forestal. Universidad Técnica Estatal de Quevedo. Quevedo, Ecuador. 45 p.

Gerderman, J., \& Nicholson, T. 1963. Spores of mycorrihizal endogene species extracted from soil by wet sieving and decanting. Transactions of the British Mycological Society, (46), 235-244.

Giovannetti, M. \& B. Mosse. 1980. An evaluation of techniques for measuring vesicular arbuscular mycorrhizal infection in roots. New Phytologist, 84, 489-500.

González, B.; Cervantes, X.; Torres, E.; Sánchez, C. \& Simba, L. 2010. Caracterización del cultivo de balsa (Ochroma pyramidale) en la provincia de Los Ríos - Ecuador. Ciencia y Tecnología, 3: 7-11.

Huang, J.; Luo, S. \& R. S. Zeng. 2003. Mechanisms of plant disease resistance induced by arbuscular mycorrhizal fungi. The Journal of Applied Ecology, 14(5):819-822.

INVAM (International Culture Collection of Arbuscular Micorrhyzal Fungi). 2016. Arbuscular micorrhyzal fungi. Key to fungi in glomales (en línea). Consultado 15 de marzo 2015. Disponible en: http://invam.wvu.edu/ 
Jiménez-Romero, E.; Garcías-Franco, L.; Carranza-Patiño, M.; Carranza-Patiño, H.; Morante-Carriel, J.; MartínezChevez, M. \& Cuasquer-Fuel, E. 2017. Germinación y crecimiento de Ochroma pyramidale (Cav. ex Lam.) Urb. en Ecuador. Scientia Agropecuaria, 8(3): 243-250.

Lekberg, Y.; Rosendahl, S. \& P. Olsson. 2015. The fungal perspective of arbuscular mycorrhizal colonization in "nonmycorrhizal" plants. New Phytologist, 205: 13991403.

MAGAP (Ministerio de Agricultura, Ganadería, Acuacultura y Pesca). 2016. Programa de incentivos para la reforestación con fines comerciales. Guayaquil, Ecuador. $71 \mathrm{p}$.

Martin, F. M.; Uroz, S. \& D. G. Barker. 2017. Ancestral alliances: Plant mutualistic symbioses with fungi and bacteria (Review). Science, 356 (6340): eaad4501. DOI: $10.1126 /$ science.aad4501.

Meza, F.; Díaz, E.; Escobar, H.; Belezaca, C.; Cachipuendo, J.; Meza, G.; López, F.; Meza, C.; Meza, J.; Cachipuendo, J. \& R. Cabrera. 2017. Identificación de Hongos Micorrízicos en Plantaciones de Melina (Gmelina arborea Roxb) en el Trópico Húmedo Ecuatoriano. Revista de Investigaciones Veterinarias del Perú. 28(4): 969-975.

Miransari, M. 2017. Arbuscular mycorrhizal fungi and heavy metal tolerance in plants. In: Wu Q.S. (eds). Arbuscular mycorrhizas and stress tolerance of plants. Springer, Singapore. 147 - 167 pp. Online ISBN 978981-10-4115-0.

Nguyen, H.; Vanclay, J.; Herbohn, J. \& J. Firn. 2016. Drivers of tree growth, mortality and harvest preferences in speciesrich plantations for smallholders and communities in the tropics. PLoS ONE, 11(10): e0164957. https://doi. org/10.1371/journal.pone.0164957.

Peterson, R.; Massicotte, H. \& L. Melvilla. 2004. Mycorrhizas: Anantomy and Cell Biology. NRC Research Press. Otawa, Canadá. 173 p.

Phillips, J. \& Hayman, D. 1970. Improved procedures for clearing roots and staining parasitic and vesiculararbuscular mycorrhizal fungi for rapid assessment of infection. British Mycological Society (55), 158-161.

Prieto-Benavides, O.; Belezaca-Pinargote, C.; Mora-Silva, W.; Vallejo-Zambrano, E.; Gutierrez-Lara, V. \& PinargoteMendoza, E. 2011. Inoculación de Brachiaria decumbens con hongos formadores de micorriza arbuscular nativos del Trópico Húmedo Ecuatoriano. Ciencia y Tecnología, 4(2): 9-18.

Prieto-Benavides, O.; Belezaca-Pinargote, C.; Mora-Silva, W.; Garcés-Fiallos, F.; Sabando-Ávila, F.; Cedeño-Loja, P. 2012. Identificación de hongos micorrízicos arbusculares en sistemas agroforestales con cacao en el trópico húmedo ecuatoriano. Agronomía Mesoamericana, 23(2):233-239.

Salazar-Zeledon, E. 2016. Influencia de altas densidades de plantación en el poder calorífico y propiedades físicas de la madera para la especie Gmelina arborea Roxb. ex Sm. Revista Forestal Mesoamericana Kurú, 13(30): 51-56.

Seguel, A.; Castillo, C. G.; Morales, A.; Campos, P.; Cornejo \& F. Borie. 2016. Arbuscular Mycorrhizal symbiosis in four Al-tolerant wheat genotypes grown in an acidic Andisol. Journal of Soil Science and Plant Nutrition, 16 (1) $164-173$

Sieverding, E.; Friedrichsen, J.; Suden, W. 1991. Vesiculararbuscular mycorrhiza in tropical agrosystems. ZB MED Nutrition Environmental Agriculture. 371 p.

Van der Heijden, M. G.; Martin, F. M.; Selosse, M. A. \& I. R. Sanders. 2015. Mycorrhizal ecology and evolution: the past, the present, and the future (Review). New Phytologist, 205: 1406-1423.

Wright, S. J. 2001. Plant diversity in tropical forests: a review of mechanisms of species coexistence. Oecologia, 130: 1-14.

Wu, Q.S. \& Y.N. Zou. 2017. Arbuscular mycorrhizal fungi and tolerance of drought stress in plants. In: Wu, Q.S. (eds). Arbuscular mycorrhizas and stress tolerance of plants. Springer, Singapore. 25 - 41 pp. Online ISBN 978-981-10-4115-0. 